ERRATA

A PREDESIGN REPORT ON MARINE WASTE DISPOSAL

VOLUME II DATA SUPPLEMENT

CITY AND COUNTY OF SAN FRANCISCO

SEPTEMBER 1971 


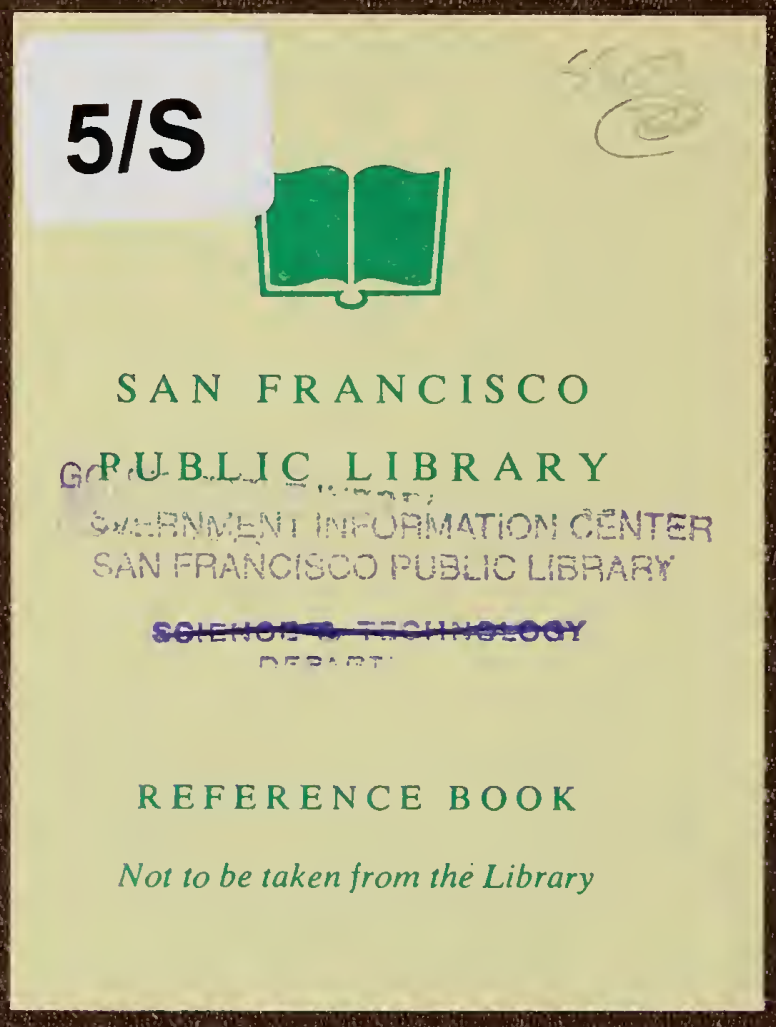




\section{DOCUMENTS DEPARTMENT}

ERRATA

A PREDESICN REPORT ON MARINE WASTE DISPOSAL

VOLUME II DATA SUPPLEMENT

CITY AND COUNTY OF SAN FRANCISCO

September 1971

$*$
$\sigma$
$N$
$\infty$
$\vdots$
$\sigma$
$\theta$
$\infty$
$N$
0
$i$
$i$

BROWN AND CALDWELL

CONSULTING ENGINEERS

SAN FRANCISCO, CALIFORNIA 


\section{$5 / S$}

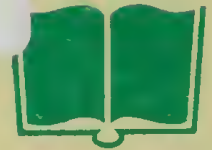

SAN FRANCISCO

GP.UB.LIC,L I B R A R Y

SX:PNA:EN! MUUGMATIOA CENTER

SAN FPANCISOO PUBLIC LIBRARY
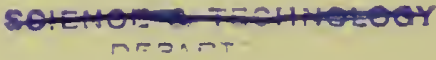

REFERENCE BOOK

Not to be taken from the Library 
ERRATA

A PREDESIGN REPORT ON MARINE WASTE DISPOSAL

VOLUME II DATA SUPPLEMENT

CITY AND COUNTY OF SAN FRANCISCO

September 1971

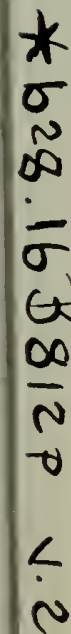

BROWN AND CALDWELL

CONSULTING ENGINEERS

SAN FRANCISCO, CALIFORNIA 


$$
\begin{aligned}
& \text { *628.16 } \\
& \text { B812 p } \\
& \checkmark .2 \\
& \text { ". } 7328
\end{aligned}
$$


The following changes are made to data sheets of Section II, Ecological Investigations, Predesign Report on Marine Wast Disposal, Volume II Data Supplement. 

BIOASSAY DATA

TYPE

In-Situ Toxicity

OBSERVER

J. Hawke

DURATION 4-8 days

SUBJECT

WATER PIER 35

ORGANISMTHREESPINE STICKLEBACK EFFLUENT

SOURCE PIER 39

GASTEROSTEUS ACULEATUS

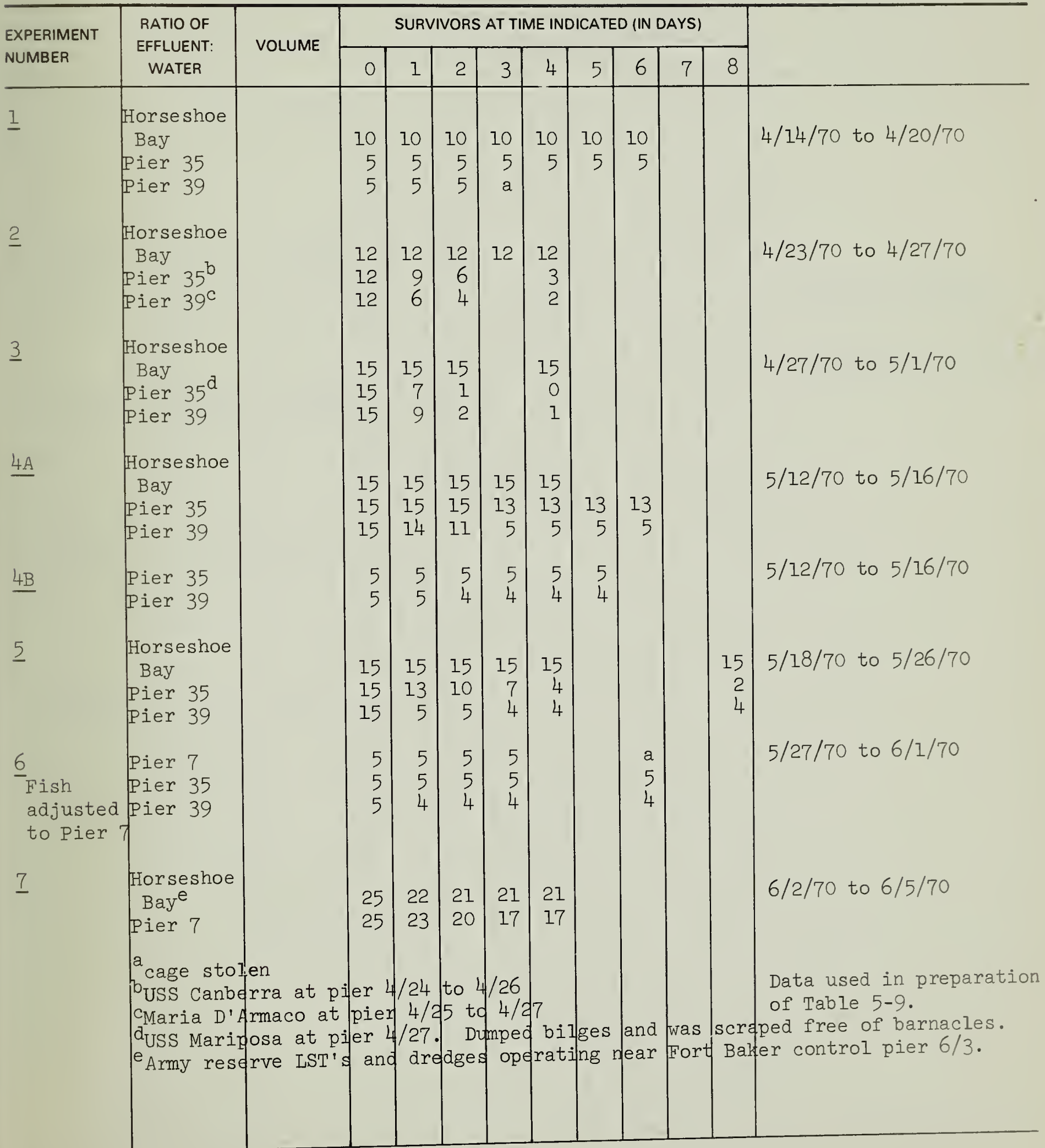




\section{Digitized by the Internet Archive in 2014}


BIOASSAY DATA

TYPE STATIC OBSERVER TRITICO DURATION 4 DAY

\section{SUBJECT} ORGANISM SHINER PERCH EFFLUENT S.F. COMPOSITE WATER SOURCE FOPT BAKER

\section{CYMATOGASTER AGGREGATA}

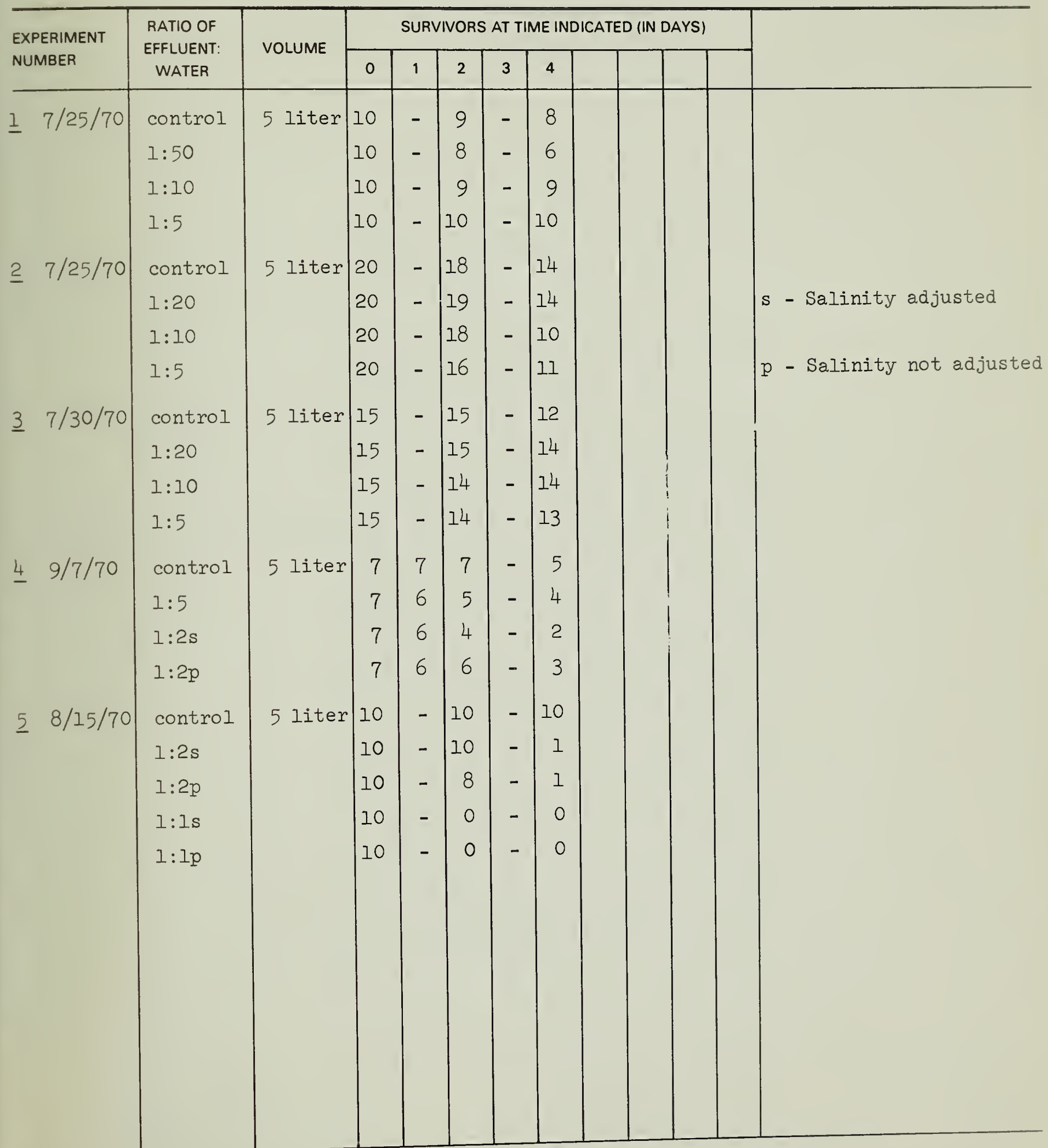



BIOASSAY DATA

TYPE STATIC OBSERVER TRITICO DURATION 4 DAY

SUBJECT ORGANISM WALIEYE PERCH EFFLUENT S.F. COMPOSITE HYPERPROSOPON ARGENTEUM
WATER SOURCE
FORT BAKER

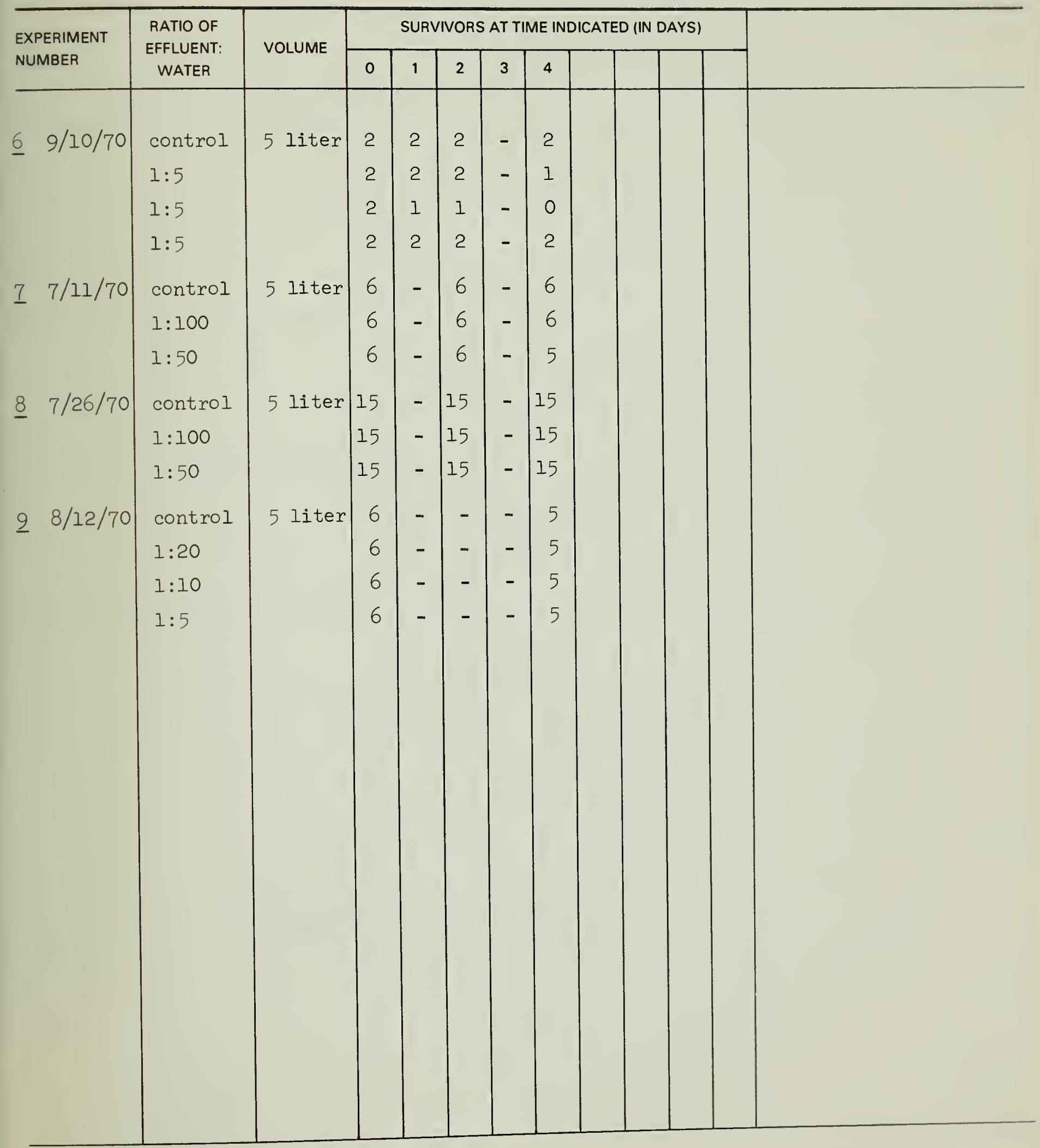



BIOASSAY DATA

TYPE

STATIC

OBSERVER

TRITICO

DURATION 4 DAY

SUBJECT

ORGANISM

SAND DAB

EFFLUENT

S.F. COMPOSITE

WATER

SOURCE

CITHARICHTHYS SORDIDUS

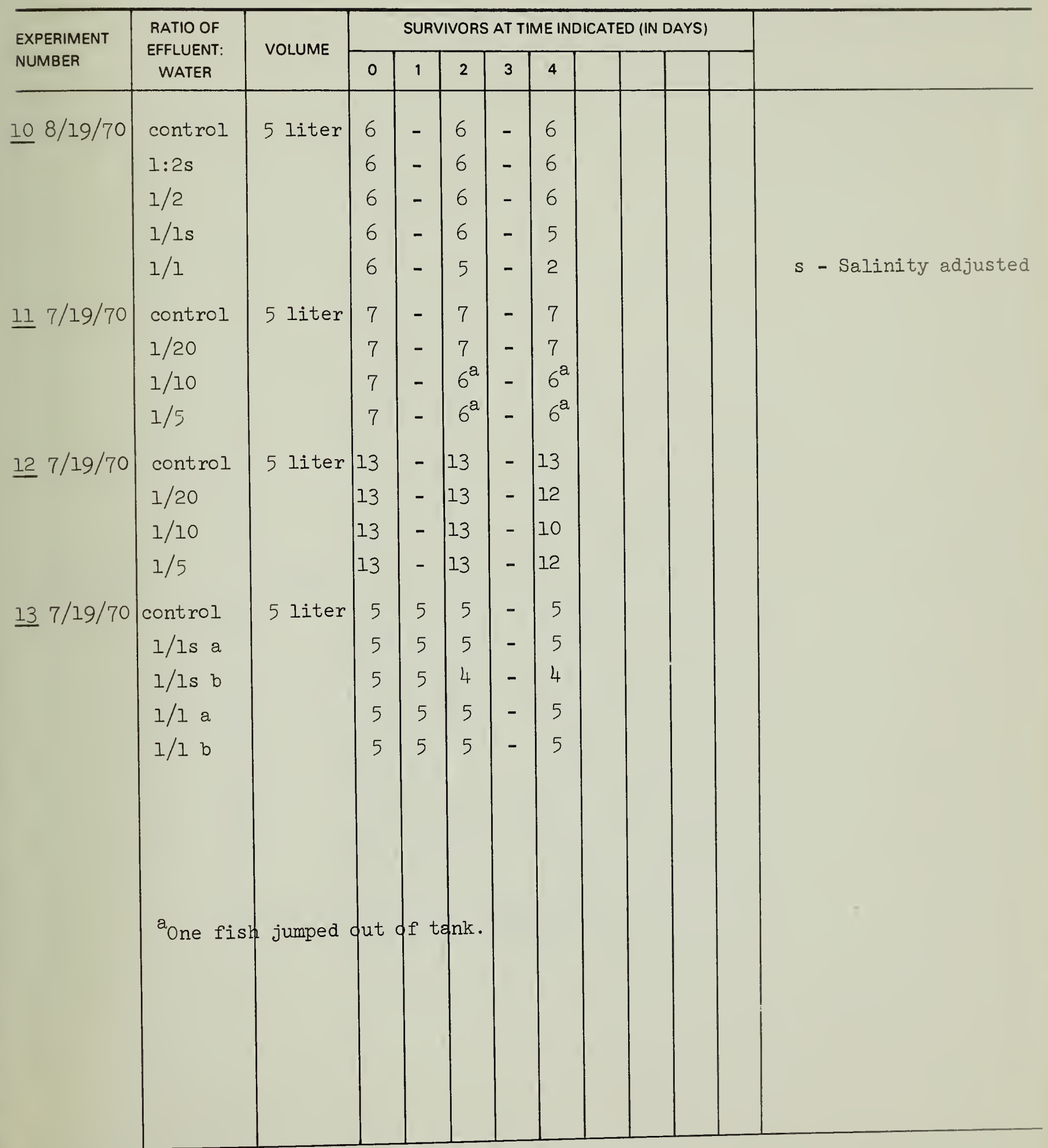



TYPE STATIC OBSERVER TRITICO DURATION 4 DAY

SUBJECT ORGANISM EFFLUENT S.F. COMPOSITE SOURCE FORT BAKER GASTEROSTEUS ACULEATUS

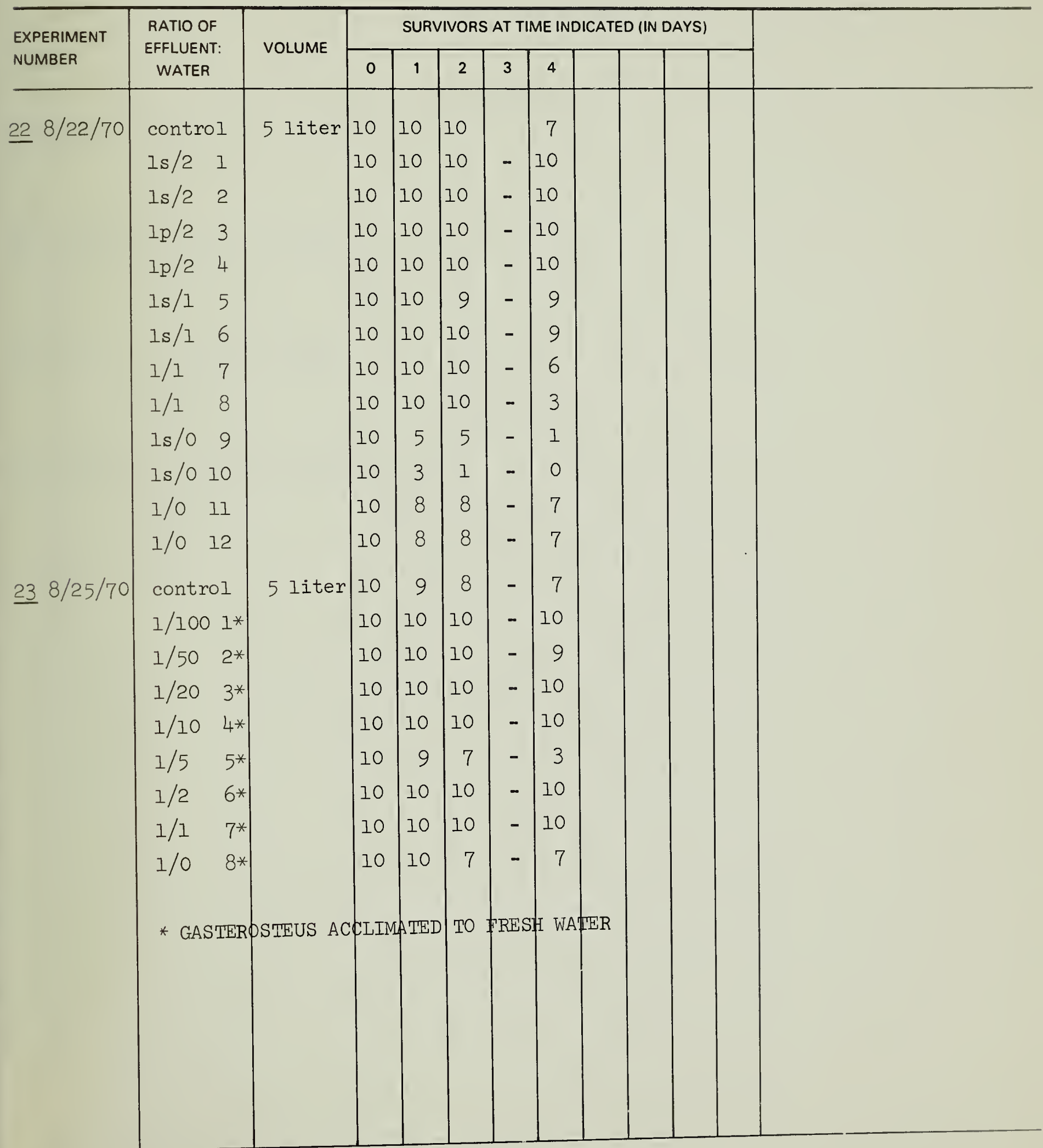



BIOASSAY DATA

TYPE

STATIC

OBSERVER

TRITICO

DURATION 4 DAY

$\begin{array}{lllll}\text { SUBJECT } & \text { HERMIT CRAB } \\ \text { ORGANISM_EFFLUENT_ S.F. COMPOSITE } & \begin{array}{l}\text { WATER } \\ \text { SOURCE }\end{array} & \text { FORT BAKER }\end{array}$

PAGURUS SAMUELIS

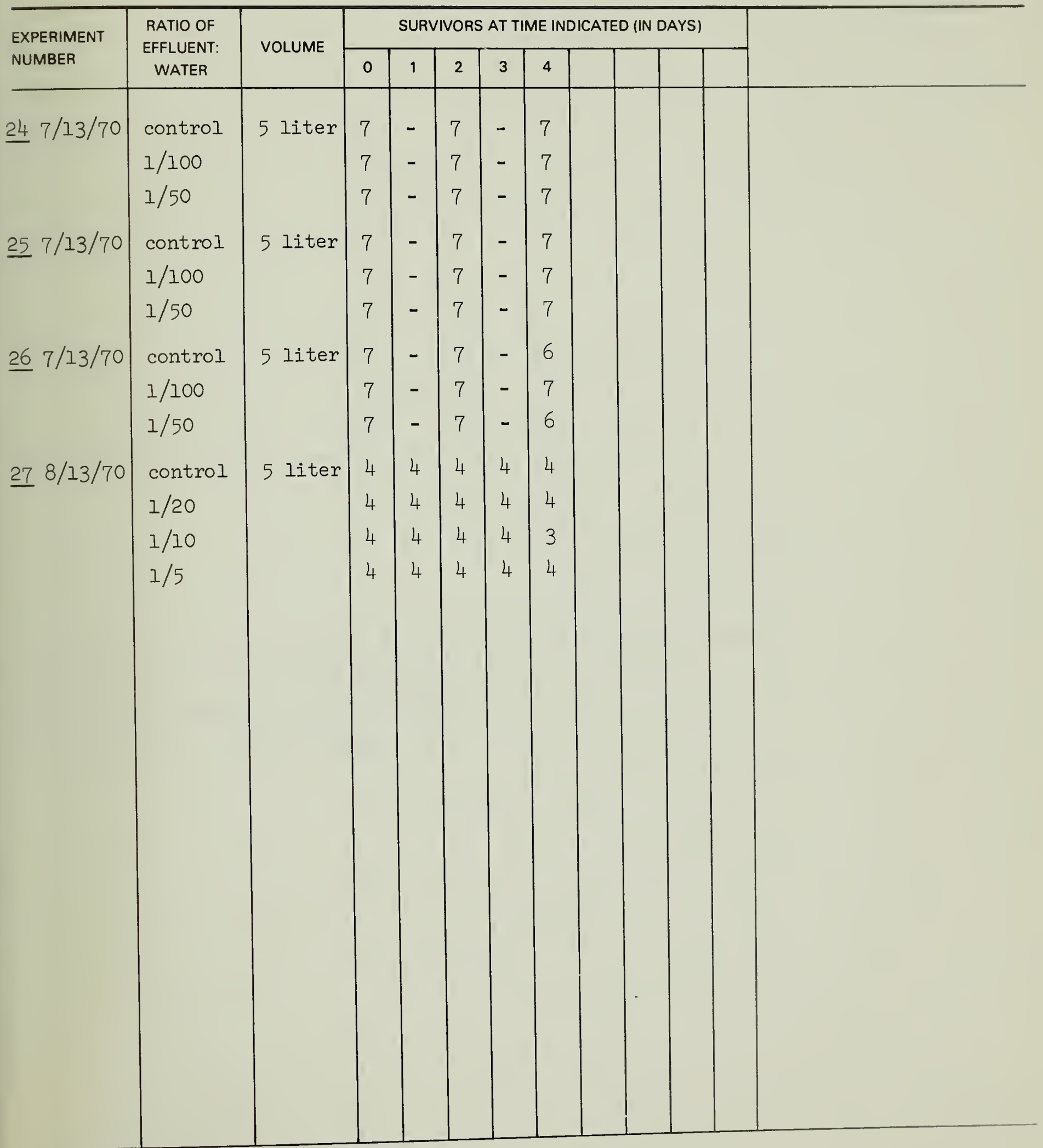




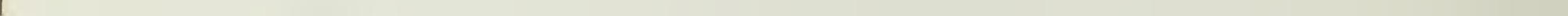


BIOASSAY DATA

TYPE STATIC OBSERVER

TRITICO DURATION 4 DAY

SUBJECT ORGANISM SHORE CRAB EFFLUENT S.F. COMPOSITE WATER SOURCE FORT BAKER TFMIGRAPSUS OREGONENSIS

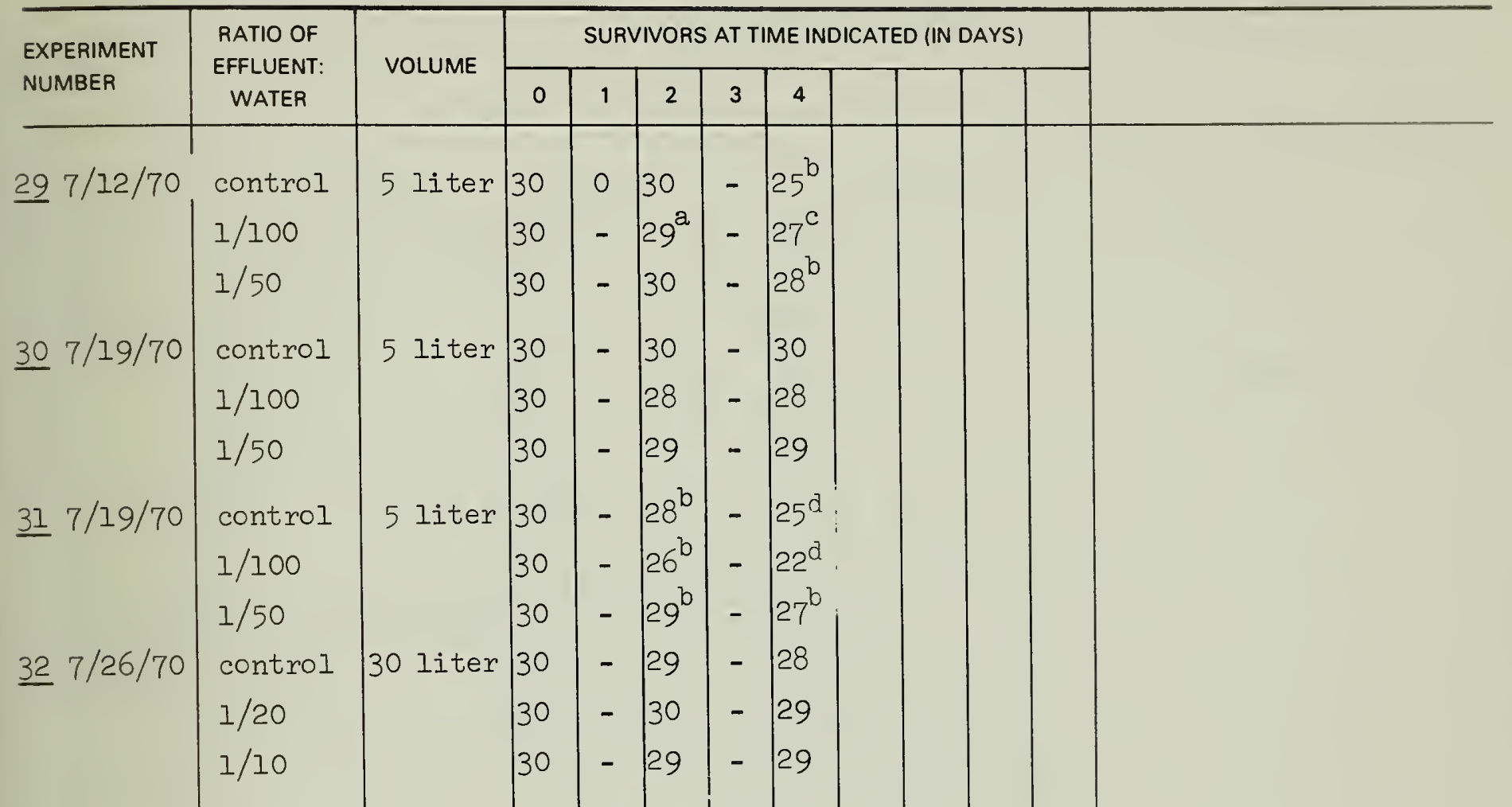

animals probably overcrowded. Filghting possibly lethal) ocqurred.

${ }^{b}$ Animals escaped - no test montalities.

Cone 48-hour mortality. Others escaped. one animal dead. others escaped. 

TYPE STATIC OBSERVER TRITICO DURATION 4 DAY

SUBJECT ORGANISM SAND CRAB EFFLUENT

S.F. COMPOSITE

WATER SOURCE FORT BAKER

EMERITA ANALOGA

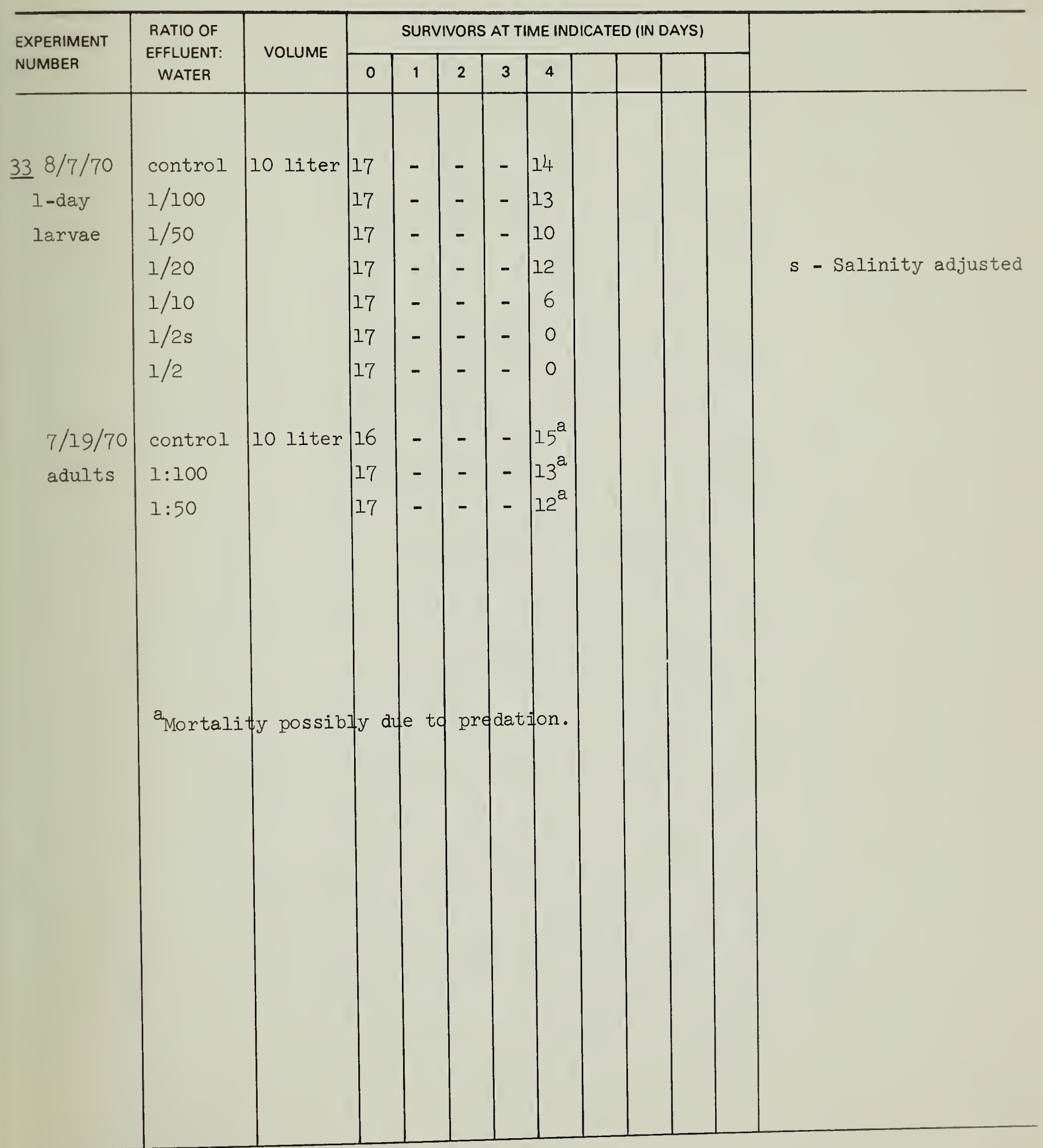



TYPE OBSERVER TRITICO DURATION 4 DAY

SUBJECT

ORGANISM BAY SHRTMP EFFLUENT

S.F. COMPOSITE WATER SOURCE FORT BAKER CRAGO SP.

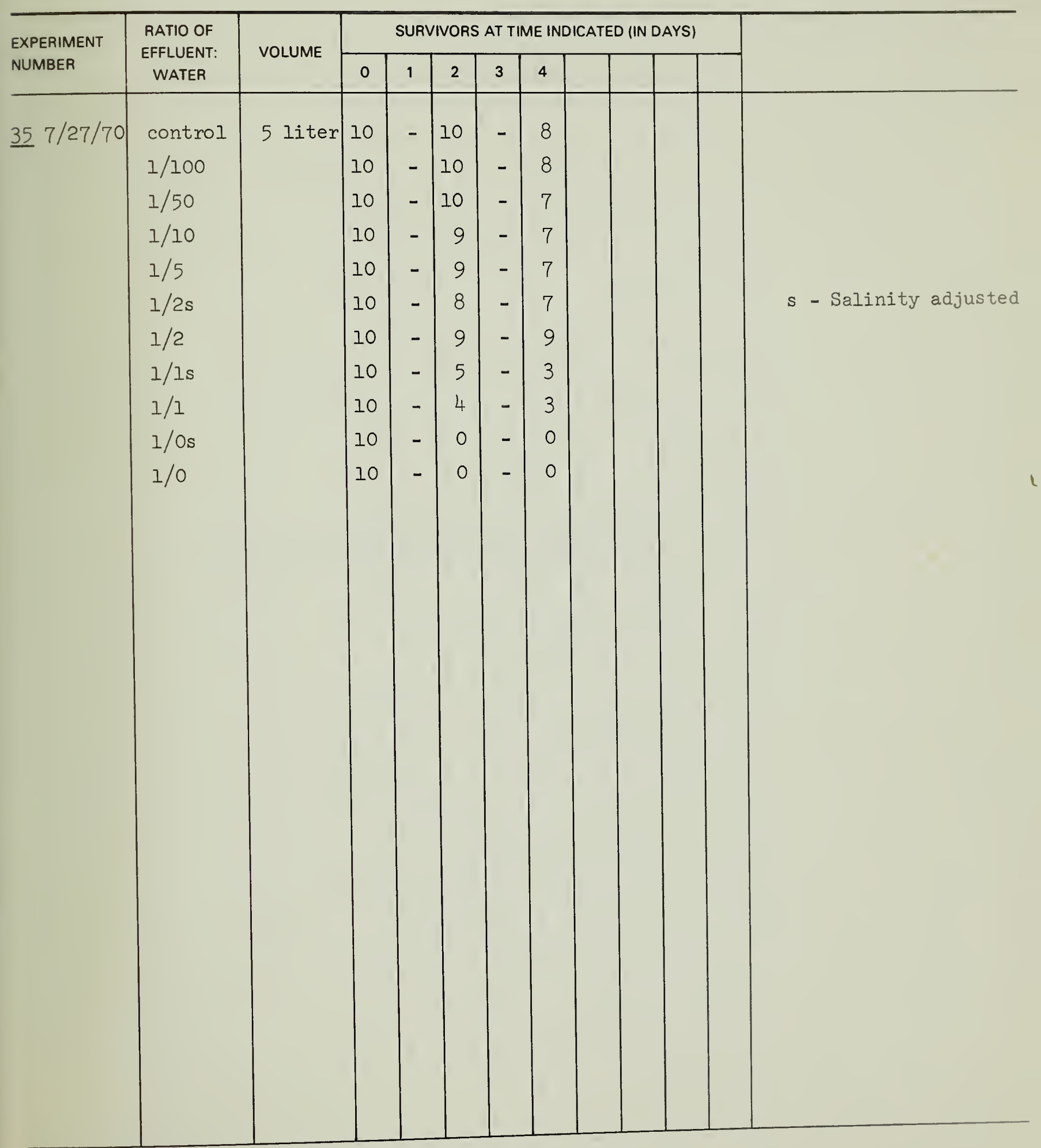



BIOASSAY DATA

TYPE OBSERVER TRITICO DURATION

4 DAY

\section{SUBJECT} ORGANISM

GHOST SHRIMP EFFLUENT

S.F. COMPOSITE

WATER SOURCE FORT BAKER

\section{CALLIANASSA CALIFORNIENSIS}

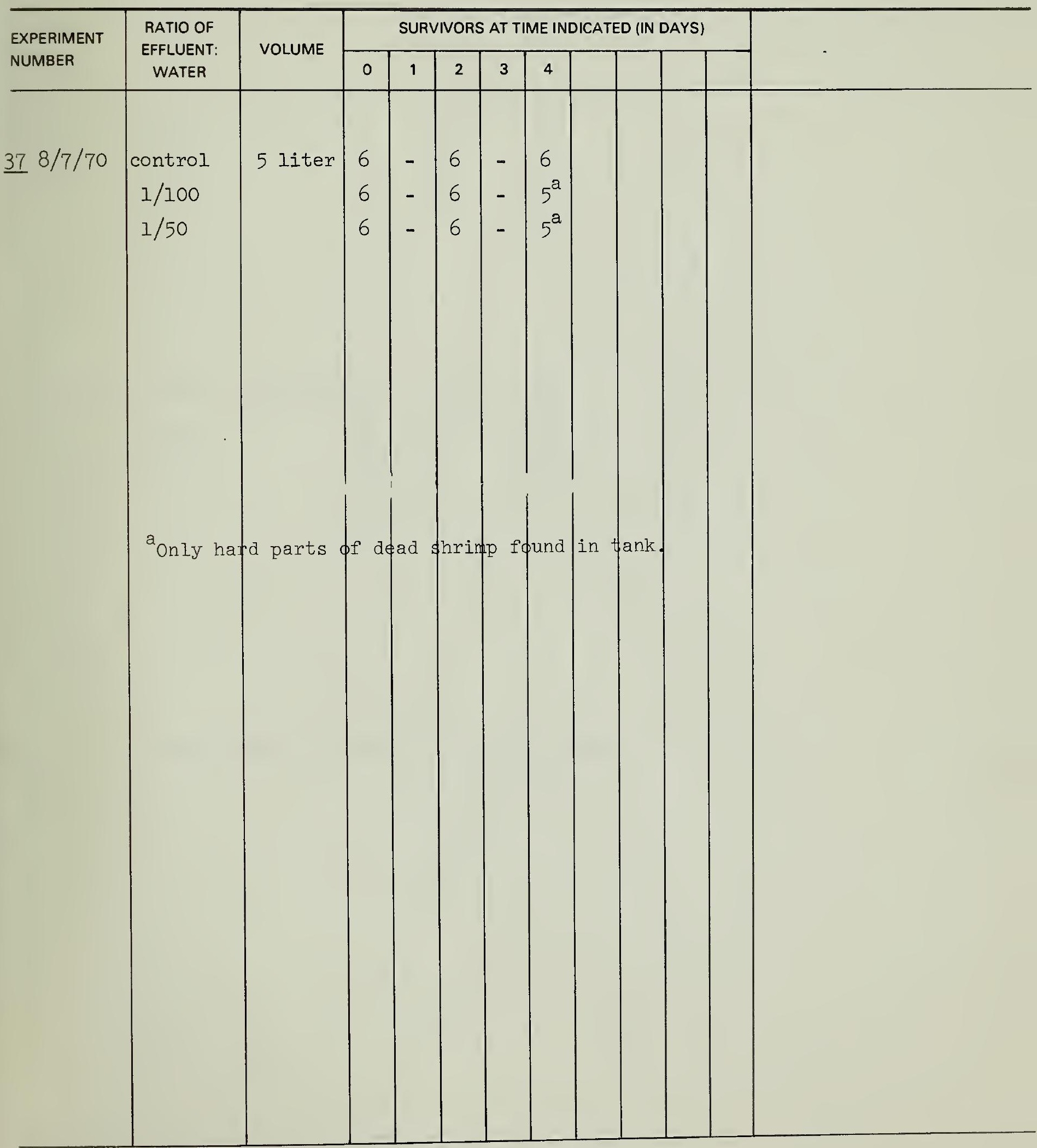


BIOASSAY DATA

TYPE OBSERVER

TRITICO DURATION 4 DAY

SUBJECT
ORGANISM_TURBAN SNAIL EFFLUENT

S.F . COMPOSITE

WATER SOURCE FORT BAKER

TEGULA FUNEBRALIS

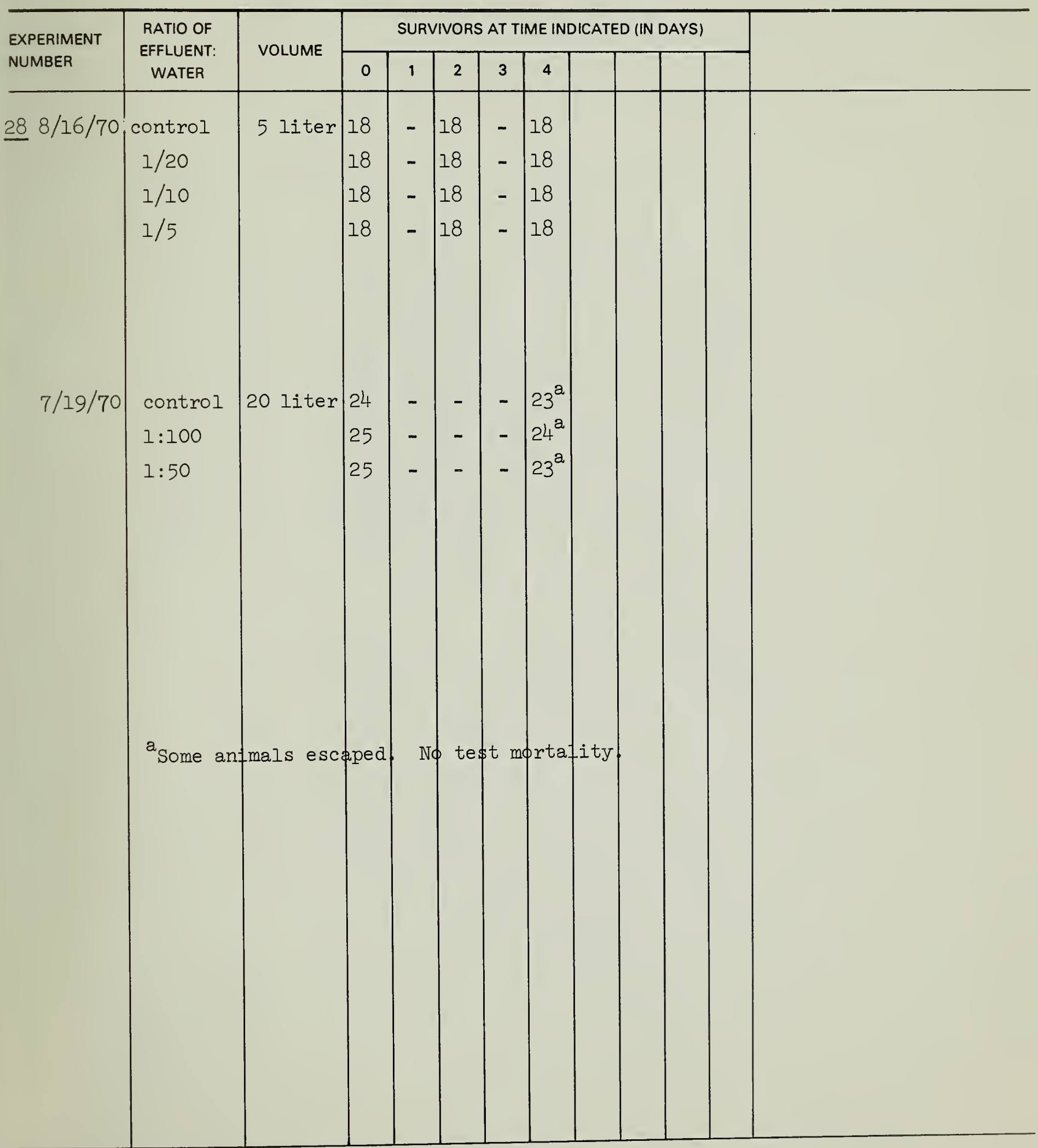



BIOASSAY DATA

TYPE STATIC OBSERVER TRITICO DURATION 4 DAY

SUBJECT ORGANISM BENT-NOSED CLAM EFFLUENT

S.F. COMPOSITE WATER SOURCE FORT BAKER

MACOMA NASUTA

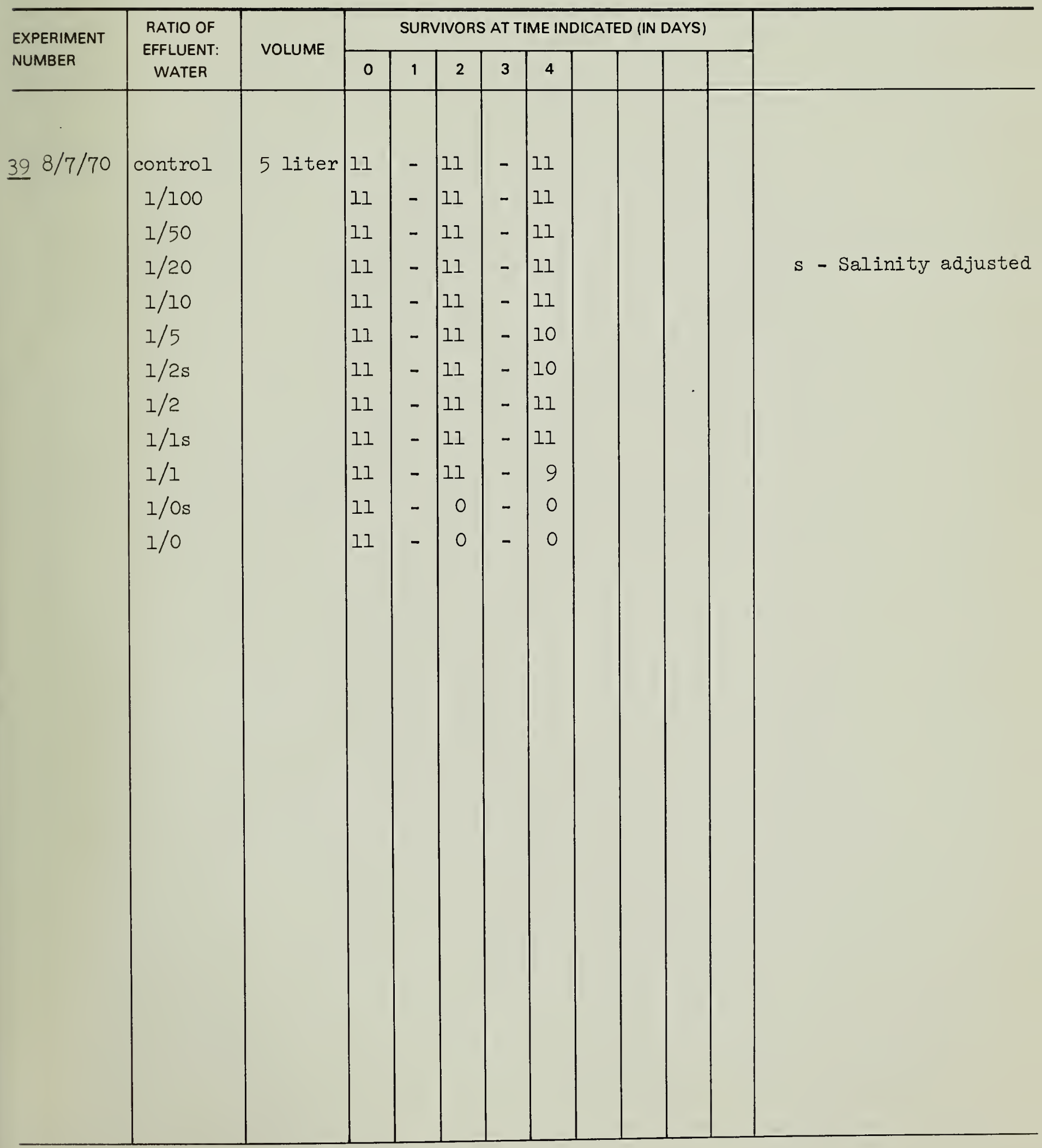



BIOASSAY DATA

TYPE STATIC OBSERVER

TRITICO DURATION 4 DAY

SUBJECT
ORGANISM_RIBBED HORSE MUSSEL EFFLUENT

S.F. COMPOSITE

WATER SOURCE FORT BAKER

VOLCELLA DEMISSA

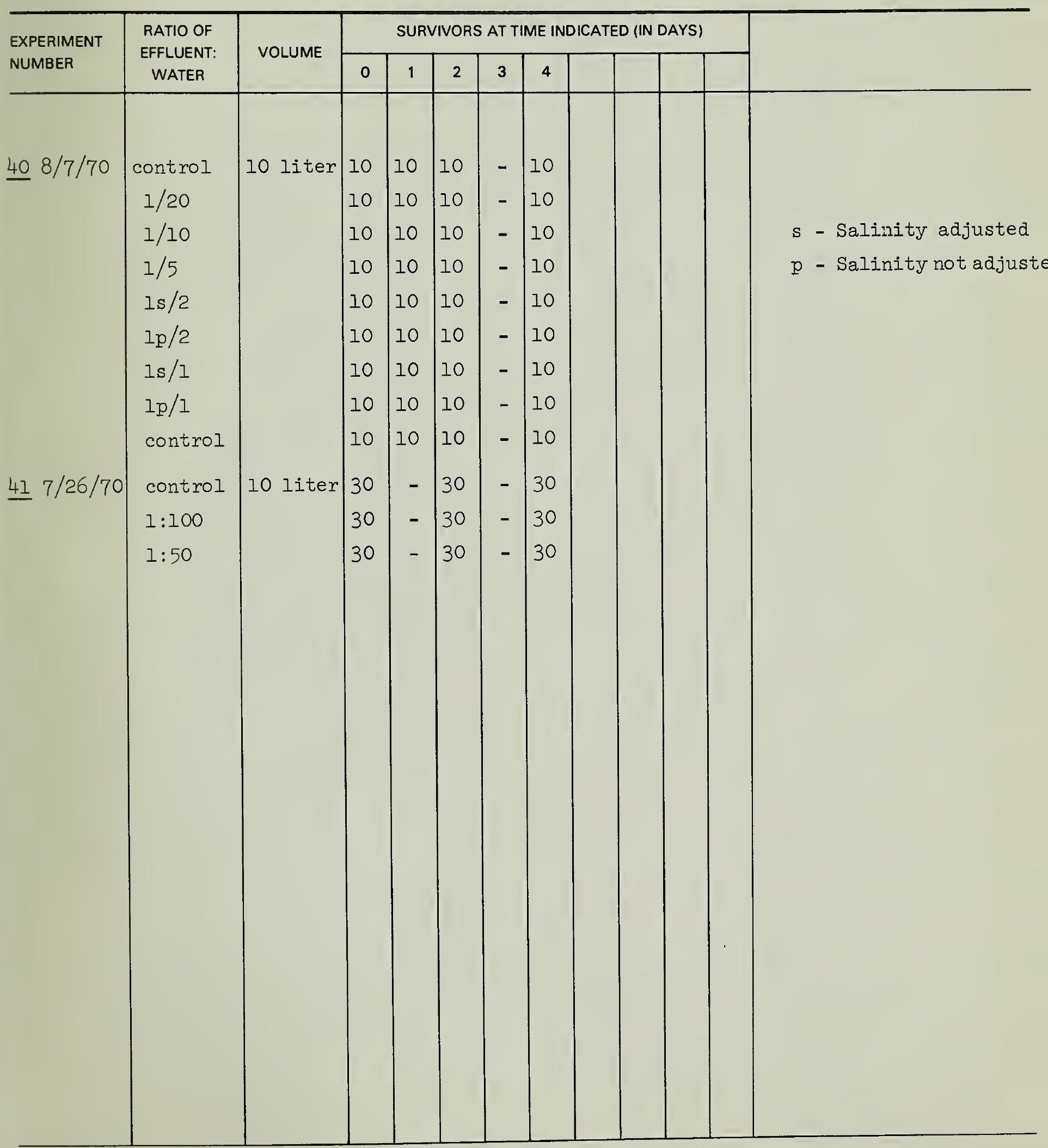





